\title{
Zur Standortbestimmung der Kontrastiven Linguistik innerhalb der vergleichenden Sprachwissenschaft ${ }^{1}$
}

\begin{abstract}
Das Programm der Kontrastiven Linguis tik wurde in den sechziger und siebziger Jahren mit der Zielsetzung formuliert, durch systematische Einbeziehung von Gemeinsamkeiten und Kontrasten zwischen Muttersprache und zu erlernender Fremdsprache den Fremdsprachenunterricht effektiver zu gestalten. Nach einigen Jahren enthusiastischer Aufnahme und Bearbeitung setzte jedoch eine allgemeine Ernüchterung und Enttäuschung ein, so dass dieses Programm eher eine bescheidene Randexistenz im Rahmen der vergleichenden Sprachwissenschaft führte und erst in den letzten Jahren unter etwas veränderten Vorzeichen wieder aufgenommen wurde. Drei Gründe waren meiner Meinung nach für diese Desillusionierung verantwortlich: (a) Die Kontrastive Linguistik wurde als Theorie des Zweitspracherwerbs gesehen und somit mit völlig unrealistischen Erwartungen verknüpft. (b) In der Erstellung der deskriptiven Grundlagen dieses Programms, d.h. in der Erstellung umfassender vergleichender Grammatiken für relevante Sprachenpaare, wurden nur wenig überzeugende Fortschritte gemacht. (c) Es fehlte eine Standortbestimmung der Kontrastiven Linguistik im Rahmen der vergleichenden Sprachwissenschaft, aus der deutlich hervorgeht, was die Möglichkeiten und Grenzen dieses Typs von Sprachvergleich sind. Nachdem sich heute die Situation bezüglich der beiden ersten Punkte erheblich verbessert hat (u.a. auch durch einschlägige Arbeiten des IDS), widme ich mich in meinem Beitrag dem dritten Punkt: Durch eine systematische Gegenüberstellung der Kontrastiven Sprachwissenschaft mit den anderen Spielarten der vergleichenden Sprachwissenschaft sollen die Erkenntnismöglichkeiten und Grenzen der verschiedenen Ansätze zum Vergleich von Sprachen bestimmt werden, so dass die Kontrastive Linguistik durch diese Gegenüberstellung klare Konturen erhält. Im Rahmen dieser Gegenüberstellung wird eine Vielzahl von Beobachtungen zum Deutschen aus der Sicht des Englischen und anderer Sprachen gemacht. Für die Kontrastive Linguistik (KL) ergibt sich abschließend das folgende Profil:
\end{abstract}

- Synchronie: Die KL ist primär synchron orientiert

- Granularität: Ihr Gegenstand sind feinkörnige Beobachtungen zu Kontrasten zwischen Sprachen

- Skopus: Die KL beschäftigt sich vor allem mit umfassenden Vergleichen von Sprachpaaren.

- Perspektivierung: Ihr Mehrwert besteht u.a. darin, dass eine Sprache aus der Perspektive einer anderen beschrieben wird. Aus dieser Perspektivenwahl ergeben sich neue Beobachtungen.

- Zielsetzung: Ihre Zielsetzung sind weitreichende, falsifizierbare Verallgemeinerungen über Kontraste. Die Wahl eines theoretischen Rahmens ist sekundär.

Für anregende Kritik und Verbesserungsvorschläge bedanke ich mich bei den Herausgebern und allen, die mit dieser Aufgabe betraut wurden. 


\section{Einleitung}

Anliegen des vorliegenden Aufsatzes ist es, den Stellenwert der Kontrastiven Sprachwissenschaft innerhalb der vergleichenden Sprachwissenschaft zu bestimmen mit der Zielsetzung, durch eine solche Standortbestimmung die Möglichkeiten und Grenzen eines bilateralen Sprachvergleichs zu erfassen. Dabei subsumiere ich unter dem Namen „Kontrastive Sprachwissenschaft" alle synchron orientierten Untersuchungen, in denen zwei, manchmal auch drei oder vier Sprachen verglichen werden, d.h. auch alle Forschungsprogramme, die unter den Bezeichnungen ,konfrontative Sprachwissenschaft", ,vergleichende Grammatik" oder "Grammatiken im Vergleich" durchgeführt werden oder durchgeführt worden sind. Somit ist auch das IDS-Projekt „Grammatik des Deutschen im europäischen Vergleich" ein wesentlicher Bezugspunkt für meine Diskussion.

Das Programm der „Kontrastiven Linguistik ${ }^{c}$ im engeren Sinn des Wortes wurde in den sechziger und siebziger Jahren des vergangenen Jahrhunderts mit der Zielsetzung formuliert, durch systematische Einbeziehung von Gemeinsamkeiten und Kontrasten zwischen Muttersprache und zu erlernender Fremdsprache den Fremdsprachenunterricht effektiver zu gestalten (vgl. Aarts 1981; Alatis (Hg.) 1968). Nach einigen Jahren enthusiastischer Aufnahme und Implementierung dieses Programms setzte jedoch eine allgemeine Ernüchterung und Enttäuschung ein, so dass die Kontrastive Linguistik eher eine bescheidene Randexistenz im Rahmen der vergleichenden Sprachwissenschaft führte und erst in den letzten Jahren unter etwas veränderten Vorzeichen und manchmal auch anderen Bezeichnungen wieder aufgenommen wurde. Drei Gründe waren meiner Meinung nach für diese Desillusionierung verantwortlich:

a) Die Kontrastive Linguistik wurde als Theorie des Zweitspracherwerbs gesehen und somit mit völlig unrealistischen Erwartungen verknüpft, die früher oder später enttäuscht werden mussten.

b) In der Erstellung der deskriptiven Grundlagen dieses Programms, d.h. in der Erstellung umfassender vergleichender Grammatiken für relevante Sprachenpaare, wurden nur wenige überzeugende Fortschritte gemacht. Umfassendere Untersuchungen zum Englischen und Deutschen z.B. (Kufner 1962) enthielten zum großen Teil allgemein bekannte, elementare Beobachtungen.

c) Es fehlte eine Standortbestimmung der Kontrastiven Linguistik im Rahmen der vergleichenden Sprachwissenschaft, aus der deutlich hervorgeht, was die Möglichkeiten und Grenzen dieses Typs von Sprachvergleich sind. 
Nachdem sich heute die Situation bezüglich der beiden ersten Punkte erheblich verbessert hat (vgl. Wienold 1973; König/Gast 2009; Hawkins/Filipović ersch.demn.), u.a. auch durch einschlägige Arbeiten des IDS, widme ich mich in meinem Beitrag ausschließlich dem dritten Punkt: Durch eine systematische Konfrontation der Kontrastiven Sprachwissenschaft mit den anderen Spielarten der vergleichenden Sprachwissenschaft sollen die Erkenntnismöglichkeiten und Grenzen der verschiedenen Ansätze zum Vergleich von Sprachen bestimmt werden. Der Raum der Diskussion lässt sich in etwa durch folgendes Diagramm abstecken:

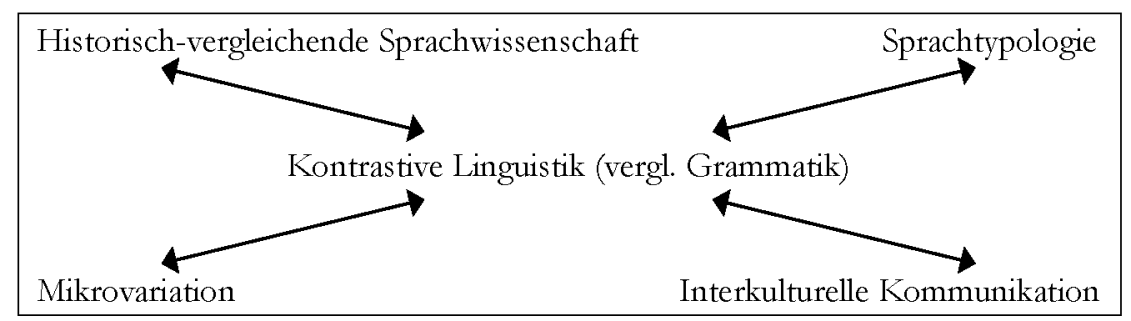

Im Rahmen dieser Diskussion soll eine möglichst große Zahl von z.T. neuen Beobachtungen über Kontraste zwischen dem Deutschen und anderen europäischen Sprachen identifiziert und kurz diskutiert werden. Detaillierte Diskussionen der angesprochenen Phänomene sind in diesem Rahmen natürlich nicht möglich.

\section{Attributive Intensifikatoren. Eine Fallstudie}

Beginnen möchte ich mit einer Fallstudie, d.h. mit einer vergleichenden Betrachtung eines winzigen Fragments der deutschen Sprache, dem Ausdruck eigen, um an diesem Beispiel zu zeigen, in welch vielfältiger Weise Eigenschaften dieses Ausdrucks durch einen Vergleich mit seinen Gegenstücken in anderen Sprachen und durch verschiedene vergleichende Perspektiven (historisch, typologisch, dialektal) deutlicher sichtbar werden, die sonst unbeachtet bleiben.

Historisch betrachtet ist der Ausdruck eigen, ebenso wie seine Gegenstücke in anderen germanischen Sprachen (engl. onm, schwed. egen etc.) ein Ausdruck für ,Besitzverhältnisse', wie auch aus den verwandten bzw. abgeleiteten Ausdrücken der Eigner, sich aneignen, Eigentum, Eigenschaften klar hervorgeht. Im Laufe seiner historischen Entwicklung hat dieser Ausdruck seinen Verwendungsradius ausgeweitet und sich, wie etwa auch der Genitiv, zu einem Ausdruck für allgemeine Zugehörigkeitsverhältnisse entwickelt, wie der Unterschied zwischen den beiden folgenden Beispielen zeigt (vgl. König/ Vezzosi 2008): 
(1) Die Behörden wollten mir mein eigenes Grundstück wegnehmen. (possessive Verwendung)

(2) Karls eigene Verluste waren viel höher als die seiner Konkurrenten. (allgemeine Zuordnung)

Gleichzeitig weisen diese beiden Beispiele auf einen Aspekt der Bedeutung von eigen hin, der mit der Charakterisierung als ,possessiv' ohnehin nicht erfasst war: auf einen emphatischen, kontrastierenden Aspekt, der auch bei der possessiven Verwendung deutlich ist. Eigen betont emphatisch die Zugehörigkeit zu dem genannten ,Besitzer ${ }^{c}$ dadurch, dass dieser (der Sprecher in (1), Karl in (2)) zu anderen möglichen (alternativen) ,Besitzern' in Opposition gesetzt wird. Diese Emphase durch Kontrastierung ist eine typische semantische Eigenschaft von Fokussierung. In (2) sind die ins Auge gefassten Alternativen (d.h. die Konkurrenten) genannt, in (1) nur implizit gegeben. Diese mit eigen verbundene Fokussierung eines ,Besitzers', ebenso wie seine Paraphrasierbarkeit durch Ausdrücke mit selbst weisen deutlich darauf, dass eigen zu den Intensifikatoren (selbst, persönlich etc.) zu rechnen ist. Wir werden darauf noch zurück kommen. ${ }^{2}$

Wenn wir einen kurzen Blick auf die syntaktischen Eigenschaften von eigen werfen, stellen wir fest, dass es pränominaler Bestandteil einer Nominalphrase sein kann und sich distributionell wie ein Adjektiv verhält. Allerdings muss eigen eine periphere Stellung in einer NP einnehmen, d.h. der Ausdruck geht anderen Adjektiven voraus und steht unmittelbar hinter einem Artikel, Quantor oder Possessorausdruck, falls vorhanden:

a. Mein eigenes neues schwarzes Auto wurde nicht beschädigt.

b. ?Mein neues, schwarzes, eigenes Auto wurde nicht beschädigt.

(4) Eine eigene abgeschlossene Wohnung ist viel wert.

Ein Vergleich mit dem Englischen zeigt, dass dort zusätzliche Restriktionen bestehen: own kann nur auf ein Possessivpronomen oder einen Genitiv folgen. Mit den Artikeln ist own nicht kombinierbar:

My own appartment ... an appartment of my own ...*an/the own appartment

Die Flexionseigenschaften von dt. eigen bestätigen seine grobe Einordnung in die Klasse der Adjektive. Der Ausdruck weist die für pränominale Adjektive charakteristischen starken und schwachen Flexionsformen auf (ein eigenes Auto - das eigene Auto). Dass Komparativ- und Superlativformen fehlen,

Diese fokussierende, kontrastierende Eigenschaft wird auch darin deutlich, dass eigen dann nicht mit Possessivpronomina kombinierbar ist, wenn ein Alternativenbezug und eine Kontrastierung ausgeschlossen sind (Karl ist groß für sein (*eigenes) Alter - Sein eigenes Alter verschweigt er gern.) 
nicht jedoch die Möglichkeit einer Graduierung durch Adverbien (etwas eigen, sebr eigen) ist als Konsequenz der Semantik des Ausdrucks zu sehen. Im Gegensatz wiederum zu seinem Gegenstück omn im Englischen kann der deutsche Ausdruck mit belebten Subjekten auch prädikativ verwendet werden:

(6) Karl ist etwas/sehr eigen.

Der Zusammenhang mit der bisherigen, attributiven Bedeutung ist allerdings nicht offensichtlich und wird erst deutlich, wenn man von der allgemeineren Bedeutung, Identität', ,Individualität' ausgeht:

(7) Karl hat seinen eigenen Kopf.

(8) Der Mann ohne Eigenschaften ...

Typologisch betrachtet gehört eigen zu den Indikatoren für Possession, aber auch zu den attributiven Intensifikatoren, d.h. zu einer Gruppe von Ausdrücken wie dt. selbst, persönlich. In vielen europäischen Sprachen können diese sowohl adnominal (der Direktor selbst) als auch adverbial verwendeten Intensifikatoren (Der Direktor wäscht sein Auto selbst.) nicht attributiv verwendet werden. ${ }^{3}$ In den Turksprachen oder im Mandarin z.B. ist dies jedoch möglich: Der gleiche Ausdruck (türk. kendi; chines. riju) entspricht sowohl dt. selbst als auch eigen in den oben genannten Sätzen. Zudem werden die genannten Ausdrücke in den beiden Sprachen auch als Reflexiva verwendet. Unter dieser vergleichenden Perspektive stellt sich auch die Frage der Beziehung von attributiv verwendetem eigen zur Reflexivität. Die folgenden Beobachtungen zeigen, dass auch im Deutschen die Beziehung zwischen dem attributiven Intensifikator und dem semantischen Bereich der Reflexivität sehr eng ist:

- Im Gegensatz zum Englischen aber parallel zum Niederländischen wird eigen neben selbst als erste Komponente in reflexiven Komposita verwendet (Selbstständigkeit - Eigenständigkeit, Selbstliebe - Eigenliebe; Selbstverantwortung - Eigenverantwontung). In der Verwendung dieser beiden Komponenten besteht eine gewisse Komplementarität, sie überlappen aber auch z.T. in ihrer Distribution, mit subtilen semantischen Konsequenzen.

- Adverbial verwendetes selbst ist durch eigen paraphrasierbar, wenn dieser Ausdruck mit geeigneten Nomina verbunden wird:

(9) Karl hat dieses Haus selbst gebaut (= mit eigenen Händen).

(10) Ich habe das mit eigenen Augen gesehen (= selbst gesehen).

3 Die hier getroffene Unterscheidung zwischen, adnominaler ${ }^{\star}$ und ,attributiver ${ }^{6}$ Verwendung ist sicherlich kein Beispiel für eine optimale terminologische Wahl und könnte auch durch die Unterscheidung, postnominal' vs. ,pränominal' ersetzt werden. 
- Eigen ist nicht nur das Gegenstïck bzw. der Lückenfüller für attributiv verwendete Intensifikatoren in anderen Sprachen, sondern wird auch verwendet, um die Unterscheidung auszudrücken, die in skandinavischen Sprachen (z.B. im Schwedischen) durch die Differenzierung von zwei Arten von Possessivpronomina ausdrückbar ist, nämlich durch die disjunkte Referenz bezeichnenden Possessivpronomen hans, sein ${ }^{6}$, hennes ,ihr', wie wir sie auch im Deutschen finden und die reflexiven Possessivpronomina, die das Deutsche nicht mehr kennt: $\sin$, sein, ihr':

(11) a. Lars $s_{i}$ tvättar hans, bil. , Lars reinigt sein Auto.' (= das Auto einer anderen Person)

b. Lars ${ }_{i}$ tvättar $\sin _{i}$ bil. , Lars reinigt sein eigenes Auto.'

Angesichts dieser Befunde eines typologischen Sprachvergleichs (vgl. König/ Gast 2006; König/Vezzosi 2008) ist es auch nicht weiter überraschend, dass Dialekte des Niederländischen (z.B. Brabants) eigen in Verbindung mit dem entsprechenden Possessivpronomen als Reflexivmarkierung verwenden:

(12) a. Hij wast zijn eigen. ,Er wäscht sich. ${ }^{c}$

b. Hij maakt hem die meesten zorgen over zijn eigen. ,Er macht sich die meisten Sorgen über sich selbst. ${ }^{c}$

Die vorgelegte Analyse von eigen zeigt, in welch vielfailtiger Weise verschiedene vergleichende Perspektiven neue Beobachtungen über eine Sprache ermöglichen, die anders kaum sichtbar würden. Das diskutierte Beispiel hat nur den Nachteil, dass es einen winzigen Ausschnitt, ein einziges lexikalisches Element mit markanten grammatischen Eigenschaften, betrifft. Im Folgenden soll daher die Diskussion systematischer, d.h. in Verbindung mit etwas größeren Sprachausschnitten geführt werden.

\section{Kontrastive Linguistik und Historisch-vergleichende Sprachwissenschaft}

Zu den Zielsetzungen der ältesten Form des Sprachvergleichs, der Historisch-vergleichenden Sprachwissenschaft, gehört die Untersuchung der genetischen Verwandtschaft zwischen Sprachen sowie die Formulierung von Regeln und,Gesetzen', die zu Veränderungen in einzelnen Mitgliedern einer Sprachfamilie geführt haben. Mit kontrastivem Sprachvergleich und der Untersuchung von Kontrasten sowie Gemeinsamkeiten hat diese Zielsetzung nur dann etwas gemeinsam, wenn es um den Vergleich von genetisch verwandten Sprachen geht. Kontraste in der Synchronie von genetisch eng verwandten Sprachen lassen sich dann oft als unterschiedliche Phasen in der Entwicklung von zwei Sprachen charakterisieren, in der eine gleichsam den Vorgaben der anderen folgt. Zwei Beispiele aus einer kontrastiven Analyse Deutsch-Jiddisch und Deutsch-Englisch sollen dies illustrieren. 


\subsection{Deiktische Richtungsadverbien}

Zum Inventar der lokaldeiktischen Ausdrücke im Deutschen - im Gegensatz zu den meisten anderen germanischen Sprachen - gehören auch die Richtungspartikeln hin und her, die u.a. als Teil des Interrogativadverbs no (woher, wohin), als Teil von komplexen, diskontinuierlichen Adpositionen, oft auch Zirkumpositionen genannt, (vom Babnhof her, zum Babnhof hin) und als Teile von zusammengesetzten Präpositionen (binauf, herauf) in Richtungsadverbien verwendet werden. Neben den genannten direktiven Komponenten gehört zum Inhalt dieser beiden Ausdrücke noch eine deiktische Komponente: hin bezeichnet eine Richtung weg vom Orientierungszentrum (Sprecherort etc.), her signalisiert die Richtung zu diesem Orientierungszentrum. In formaler, geschriebener Sprache ist diese interessante Unterscheidung des Deutschen durchaus lebendig und als Norm anzusehen. In informeller, gesprochener Sprache finden wir jedoch in zunehmendem Maße eine Neutralisierung dieser Unterscheidung durch den Ausdruck , $r+$ Präposition' (hinibler/heriber > ribber). ${ }^{4}$ Diese Entwicklung ist allgemein bekannt, zumal sie Gegenstand vieler sprachkritischer und sprachpflegerischer Anmerkungen ist. Was hier in besonderem Maße interessiert, ist die Tatsache, dass im Jiddischen eine analoge Entwicklung schon vor vielen Jahrhunderten eingetreten sein muss (vgl. (14)):

\section{DEUTSCH}

(13) a. Wir schwimmen jetzt über den Fluss. - Wir schwimmen jetzt hinüber zu dir.

b. Ich bin auf der anderen Seite des Flusses. Schwimm doch herüber zu mir.

c. Wir schwimmen jetzt rüber. - Schwimm doch rüber.

d. Geh doch rein/hinein. - Komm doch rein/herein.

JIDDISCH (wesentlich frühere Entwicklung)

(14) a. zi geyt arayn un er geyt aroys. ,Sie geht rein und er geht raus.c

b. aroyf , rauf ${ }^{6}$, arieber, rüber $^{6}$

Ohne dass hier in irgendeiner Weise eine Kausalbeziehung unterstellt werden soll, kann man also sagen, dass aktuell eine Vereinfachung im System der Lokaldeixis im Deutschen zu beobachten ist, die im Jiddischen schon viel früher stattfand, dass also das Deutsche den Vorgaben des Jiddischen folgt. Eine solche vergleichende Betrachtung dieser Entwicklung ist auch insofern von besonderem Interesse, als die Unterscheidung zwischen ,Ort ${ }^{c}$ und ,Richtung ' nicht nur in den romanischen Sprachen weitgehend fehlt, sondern auch im Englischen verlorenging. Während noch im Frühneueng-

Auf wichtige Einzelheiten dieser Entwicklung wird hier nicht näher eingegangen: So ist diese Neutralisierung nur dann möglich, wenn die Präposition mit einem Vokal beginnt (raus vs. bervor), in wird durch ein ersetzt (rein) etc. 
lischen, also bei Shakespeare, Differenzierungen wie die zwischen whence ,woher ${ }^{c}$ und whither , wohin' ${ }^{c}$ gebräuchlich waren, werden Reste analoger Oppositionen (hence, von da her/folglich - bither, hierher') im heutigen Englisch höchstens noch metaphorisch oder in idiomatischen Ausdrüicken gebraucht. Eine generelle Unterscheidung zwischen, Ort ${ }^{c}$ und ,Richtung fehlt im Englischen fast völlig und ergibt sich interpretatorisch lediglich aus der Verbsemantik und dem Aspekt, wie die folgenden kontrastierenden Beispiele belegen:

(15) a. (engl.) John went outside. He is still outside.

b. (dt.) Karl ist raus/nach draußen gegangen. Er ist immer noch draußen.

c. (engl.) John went upstairs. He is still upstairs.

d. (dt.) Karl ist nach oben gegangen. Er ist immer noch oben.

\subsection{Konditionalsätze mit Inversion}

Ähnliche Beobachtungen über Phasenverschiebungen analoger historischer Prozesse wie sie eben für das Jiddische und Deutsche gemacht wurden, lassen sich auch besonders häufig für das Deutsche und das Englische machen. Ein bekanntes Beispiel ist die teilweise parallele Entwicklung der Präterito-Präsentia in den beiden Sprachen: Die Entwicklung dieser Teilklasse von Verben zu Hilfsverben, die im Englischen nicht mehr allein das Prädikat eines Satzes bilden können und weitgehende Lücken und Idiosynkrasien in ihren Flexionssystemen aufweisen, ist im Deutschen ähnlich verlaufen, aber weniger weit fortgeschritten. Während im Frühneuenglischen noch weitgehende Parallelen zum heutigen Deutsch zu finden sind, ist im Englischen diese Entwicklung zu einer völlig separaten Klasse von Hilfsverben erheblich weiter gegangen als im Deutschen (vgl. Plank 1984a):
a. (dt.) Ich muss jetzt weg.
b. (frühneuengl.) I must away.
c. (engl.) I must go (away).

Bei einem weiteren Beispiel, das hier etwas näher beleuchtet werden soll, handelt es sich auch um eine Besonderheit der germanischen Sprachen, nämlich um Konditionale, die durch Erststellung des Verbs, bzw. durch Inversion von Verb und Subjekt als solche gekennzeichnet sind. Was die Genese dieser Konditionalstrukturen anbelangt, so ist die Annahme sehr plausibel, dass sie durch Grammatikalisierung von Diskursen mit Interrogativsätzen entstanden sind (van den Nest 2010). Damit wäre zunächst einmal die Tatsache erklärt, dass die durch Verberststellung gebildeten Konditionalsätze eine spezifische Erscheinung germanischer Sprachen sind, ebenso wie die entsprechenden Interrogativsätze, die nach dem jetzigen Stand un- 
seres Wissens vor allem in Europa und vor allem in germanischen Sprachen zu finden sind (vgl. Haspelmath et al. (Hg.) 2005, S. 470). Nach einer in van den Nest (2010) detailliert entwickelten und durch historische Daten dokumentierten These entwickelten sich die Verberst-Konditionale aus Frageund Antwortstrukturen, in denen eine positive Antwort gleichsam vorweggenommen war, d.h. in etwa nach dem folgenden Schema:
a. Interessieren Sie sich für Urlaub in der Karibik? - Ja. - Dann zeige ich Ihnen unseren Katalog.
b. Interessieren Sie sich für Urlaub in der Karibik, dann zeige ich Ihnen unseren Katalog.

Im heutigen Englisch sind solche Konditionale marginal und nur noch in konservativem, formellem Sprachgebrauch anzutreffen. Vor allem aber gilt für die Varietäten und Register, in denen sie überhaupt noch anzutreffen sind, die Restriktion, dass nur noch drei modale Hilfsverben - bzw. Formen von modalen Hilfsverben - diese Inversionen zulassen: had, should und were:

\section{ENGLISCH}

(18) a. Had I known this I would have acted earlier. ,Hätte ich das gewusst, hätte ich früher gehandelt. ${ }^{c}$

b. Should he come earlier we might be able to go to the movies. ,Sollte er früher kommen, könnten wir ins Kino gehen. ${ }^{c}$

c. Were he to reveal his secrets there would be a major scandal. ,Würde er die Geheimnisse lüften, gäbe es einen großen Skandal.

Im Deutschen sind solche Konditionalstrukturen sicherlich weit weniger Beschränkungen unterworfen als im Englischen, auf ihren altmodischen, formellen Charakter ist aber immer wieder hingewiesen worden (vgl. Reis/ Wöllstein 2010). Außerdem kann man, auch ohne die entsprechenden empirischen Untersuchungen durchzuführen, behaupten, dass auch im Deutschen in solchen Konditionalstrukturen eine Präferenz für Hilfsverben zu beobachten ist. In Beispielen wie den folgenden haben meine Beobachtungen und Befragungen eine klare Präferenz für die Beispiele unter (a) ergeben:

\section{DEUTSCH}

(19) a. Hätte ich das gewusst ...; Sollte er früher kommen ...; Würde er regelmäßig Sport treiben ...; Könnte ich mich dagegen wehren ...; Wäre das mein Hund ...;

b. Triebst du regelmäßig Sport, dann wärest du gesünder. Nimmst du teil, dann komme ich auch. Reiste er um 8 Uhr ab, dann müsste er zu Mittag hier sein.

Ebenso wie im Falle der Neutralisierungen von Richtungsadverbien gewinnt man also auch im vorliegenden Fall den Eindruck, dass das Deutsche mit zeitlicher Verzögerung einen Prozess des Wandels durchläuft, der in ande- 
ren germanischen Sprachen in zumindest ähnlicher Form schon wesentlich früher stattfand. Bei der Untersuchung von Phänomenen dieser Art überschneiden sich die Interessen von Historisch-vergleichender Sprachwissenschaft und Kontrastiver Linguistik und ein detaillierter synchroner Vergleich kann zum Anstoß und Ausgangspunkt wesentlicher historischer Wandelprozesse in genetisch verwandten Sprachen werden. In der Niederlandistik haben solche Untersuchungen eine lange Tradition. Der Versuch von van Haeringen (1956), die strukturellen Eigenschaften des Niederländischen systematisch auf Entwicklungslinien zwischen dem Deutschen und dem Englischen zu bestimmen, hat eine Fülle analoger oder zumindest ähnlicher Wandelprozesse in den drei Sprachen identifiziert, die allerdings jeweils unterschiedliche Stufen erreicht haben. Die von dieser Arbeit ausgehenden Impulse sind gerade in den letzten Jahren systematisch weiterverfolgt worden (vgl. Hüning et al. (Hg.) 2006).

Ein bisher wenig beachtetes Phänomen soll hier noch zur weiteren Illustration angefügt werden. Die Möglichkeit, durch das Präfix be- applikative Verben zu bilden und Argumente sowie Adjunkte nahezu aller semantischer Rollen zu direkten Objekten zu machen, war ursprünglich Teil der Wortbildungsregeln aller drei westgermanischen Sprachen. Das Deutsche hat diesen mit Valenzveränderungen verbundenen Wortbildungstyp weiter verstärkt, so dass im heutigen Deutsch nicht nur präpositionale Ergänzungen, sondern in marginalen Fällen auch Adjunkte (freie Angaben) zu direkten Objekten gemacht werden können:

(20) a. Karl stieg auf den Berg. - Karl bestieg den Berg.

b. Wir sprachen über das Problem. - Wir besprachen das Problem.

c. Wir sollten mal auf dem neuen Parkett tanzen. - Wir sollten mal das neue Parkett betanzen.

Im Englischen sind durch das be-Präfix eingeleitete Verben (behold, behave, betray, beseech, belabor, belie, betoken, benumb etc.) selten, gehören oft einer sehr gehobenen Stilebene an und sind nur noch in ganz wenigen Fällen kompositionell von einem Basisverb oder Nomen und einem applikativen Präfix ableitbar:

(21) a. John moaned about his fate - John bemoaned his fate.

b. John is friends with his neighbors. - John befriends his neighbors.

Bei diesem Wortbildungsprozess, wie in vielen anderen Fällen, nimmt das Niederländische eine Position zwischen den beiden anderen westgermanischen Sprachen ein: Im Niederländischen gehört die Ableitung von applikativen Verben noch zu den produktiven Wortbildungsprozessen, ist aber wesentlich stärker eingeschränkt als im Deutschen (z.B. derven - bederven, dienen - bedienen, denken - bedenken, werken - bewerken etc.). 


\section{Kontrastive Linguistik und Sprachtypologie}

Ziel der Sprachtypologie ist es, Raum und Grenzen der Variation zwischen den Sprachen der Welt auszuloten. Dabei geht sie von der Annahme aus, dass Variation zwischen den Sprachen weder beliebig noch unbegrenzt möglich ist. ${ }^{5}$ Obwohl synchron orientiert, ist Sprachtypologie in ihrem Radius weder geografisch noch zeitlich eingeschränkt und verfolgt, etwas vereinfacht ausgedrückt, das Ziel, Muster der Variation (Sprachtypen) und Grenzen der Variation ( $\rightarrow$ Universalien) zu bestimmen. Diese absoluten oder statistischen Universalien bzw. universellen Tendenzen haben typischerweise die Form von Implikationszusammenhängen (,Wenn eine Sprache die Eigenschaft A hat, hat sie auch die Eigenschaft B) und von Ketten solcher Implikationen bzw. Hierarchien der Form (22), die folgende Verallgemeinerung zum Ausdruck bringt: Wenn eine Sprache auf irgendeiner Position P die Eigenschaft $\mathrm{E}$ hat, dann hat sie diese Eigenschaft auch auf allen Positionen links von P. Ein allgemein bekanntes Beispiel für eine solche Hierarchie ist die von Faltz (1985) für die Interaktion von Person und der Verfügbarkeit eines speziellen Reflexivpronomens formulierte Hierarchie (22b):

$$
\begin{array}{ll}
\text { a. } & \mathrm{A}>\mathrm{B}>\mathrm{C}>\mathrm{D}>\mathrm{E} \\
\text { b. } & 3>2>1 \text { (Interaktion von Reflexivität und Person) }
\end{array}
$$

Während manche Sprachen, wie z.B. das Englische (myself, yourself, him-/ berself etc.), Jiddische ( $z i k b)$ oder Russische (sebja) spezielle, von den Personalpronomina unterschiedene Reflexiva haben, ${ }^{6}$ gibt es solche Reflexivpronomina im Deutschen nur für die 3. Person (sich), während in der ersten und zweiten Person die Personalpronomina auch zum Ausdruck von Koreferenz verwendet werden (Karl hasst sich/ihn. vs. Ich hasse mich, Karl hasst mich).

Was die Kontrastive Linguistik mit der Sprachtypologie verbindet, ist vor allem die synchrone Orientierung, was sie trennt, ist der Umfang des Untersuchungsbereichs. Kontrastive Untersuchungen sind auf zwei, drei oder vier Sprachen beschränkt, Sprachtypologie ist dem Anspruch nach allumfassend, wenn auch in der Praxis auf eine repräsentative Stichprobe von Sprachen beschränkt. Aus diesem fundamentalen Unterschied ergeben sich weitere unterschiedliche Möglichkeiten und Grenzen. Die Beschränkung der Kontrastiven Linguistik auf zwei oder zumindest einige wenige Sprachen schafft die Möglichkeit für umfassende Vergleiche dieser Sprachen entlang vieler Parameter der Variation, für feinkörnige Untersuchungen und damit letztlich für eine Annäherung an die traditionelle - heute weitgehend

$5 \quad$ Während diese Prämisse in den letzten fünf Jahrzehnten fester Ausgangspunkt für umfassende sprachtypologische Programme war, ist sie in jüngster Zeit durch Evans/Levinson (2009) in Frage gestellt worden.

Im Russischen und Jiddischen werden die spezifischen Reflexiva allerdings undifferenziert für alle Personen verwendet, sind aber immer von den Personalpronomina unterschieden. 
aufgegebene - Zielsetzung, eine holistische Typologie oder Charakterologie für eine Sprache zu formulieren (vgl. Hawkins 1986; König 1996). Die angestrebten Erklärungen für die Befunde der beiden Typen des Sprachvergleichs müssen auch völlig unterschiedlich ausfallen. Kontraste zwischen einzelnen Sprachen können als Folgen von genetischer Herkunft, von historischen Prozessen und von Sprachkontakt erklärt werden. Erklärungen in der empirisch orientierten Sprachtypologie dagegen können sich auf funktionale bzw. kognitive Prinzipien wie Ikonizität, Ökonomie, Häufigkeit, konkurrierende Motivation etc. berufen. Allerdings können sich auch die beiden Spielarten des Sprachvergleichs wechselseitig befruchten: Die Kontrastive Linguistik ist auf eine allgemeine Einordnung einzelsprachlicher Phänomene in einen typologischen Rahmen angewiesen und kann aber ihrerseits, ausgehend von Einzelbeobachtungen, umfassende typologische Untersuchungen anstoßen. So lässt sich insbesondere bei aktuellen hoffnungsvollen Ansätzen zu einer semantischen oder lexikalischen Typologie (Koptjevskaja-Tamm/Vanhoeve/ Koch 2007; Vanhoeve (Hg.) 2008; Evans 2011) zeigen, wie sehr diese Arbeiten durch feinkörnige kontrastive Untersuchungen angestoßen wurden. Die Beobachtung, dass eine Sprache wie das Deutsche systematisch zwischen effizierten und affizierten Objekten durch Verbpräfixe differenziert, während dies im Englischen und in den romanischen Sprachen nicht der Fall ist (vgl. Plank 1984b), kann zum Ausgangspunkt für umfassendere typologische Studien gemacht werden (vgl. Stiebels 1996). Die folgenden Beispiele illustrieren die erwähnten Kontraste:

(23) a. ein Bild malen (effiziertes Objekt) - eine Wand bemalen (affiziertes Objekt)

b. (engl.) to paint a picture - to paint a wall

c. einen Streit vermeiden (effiziertes Objekt) - einen Streit meiden (affiziertes Objekt)

d. (engl.) to avoid a quarrel (Übersetzung für beide dt. Ausdrücke); (frz.) éviter

(24) (frz.) siffler la Marseillaise - , die Marseillaise pfeifen' (effiziert) oder ,die Marseillaise auspfeifen' (affiziert)

Ebenso bauen die umfassenderen Untersuchungen von Koch (vgl. Koptjevskaja-Tamm/Vanhoeve/Koch 2007) zu Körperteilen (dt. Haar - frz. cheveux, poile) oder zum Sinnbezirk, Wald' (dt. Hol, Baum, Wald) im Wesentlichen auf kontrastiven Untersuchungen auf.

\subsection{Artikel und Artikelgebrauch}

Die folgende Illustration unterschiedlicher Fragestellungen, Möglichkeiten und Ergebnisse der beiden diskutierten Spielarten vergleichender Sprachwissenschaft beginnt mit einem sehr begrenzten Sprachausschnitt, der Frage 
der Verfiigbarkeit eines Artikelsystems in Sprachen, d.h. der Opposition zwischen einem indefiniten und einem definiten Artikel. Die historischen Quellen dieses grammatischen Subsystems sind gut bekannt, ebenso wie die Tatsache, dass diese Kategorie vor allem im Kernbereich europäischer Sprachen (mit Ausnahme der meisten slawischen und finno-ugrischen Sprachen) zu beobachten ist, und dass an der Peripherie der Sprachen mit Artikelsystemen, insbesondere im Sorbischen und im Finnischen, entsprechende Kategorien gebildet werden (vgl. Heine/Kuteva 2005). Diese Kategorie des Artikels als Manifestation eines europäischen Sprachbundes zu bezeichnen, ist allerdings angesichts ähnlicher Subsysteme in ozeanischen Sprachen nicht ganz angemessen.

Die Frage der Existenz bzw. Nicht-Existenz eines Artikelsystems in einer Sprache ist somit ein interessanter Parameter der Variation in der Struktur von Nominalphrasen, für kontrastive Analysen jedoch nur der Ausgangspunkt von weitergehenden Fragen, wie z.B. den folgenden: Werden bestimmte Artikel in zwei kontrastierten Sprachen in unterschiedlichen Kontexten (z.B. mit Eigennamen, mit Abstrakta etc.) verwendet? Welche Konstruktionen entsprechen in artikellosen Sprachen der Opposition ,definiter - indefiniter Artikel ${ }^{c}$ in Sprachen mit Artikeln? Wie interagieren Artikel mit anderen nominalen Kategorien wie Genus, Numerus? Über alle diese Fragen lassen sich in europäischen Sprachen interessante Beobachtungen machen. So werden z.B. im Griechischen Eigennamen stets mit dem definiten Artikel verbunden, während dies im Deutschen (die Ferres, der Peter) mit speziellen Bedeutungsnuancen möglich, aber nicht obligatorisch ist. Im Englischen sind solche Kombinationen - mit Ausnahme von Verbindungen mit restriktiven Relativsätzen (The Mary I have in mind ...) - völlig ausgeschlossen. Bei der Kombinierbarkeit mit Abstrakta stehen einer obligatorischen Verwendung des definiten Artikels im Französischen ( $I$ a solitude n'existe pas) die wahlweise Verwendung im Deutschen ((Die) Einsamkeit ist schwer zu ertragen) und die artikellose Verwendung von Abstrakta im Englischen gegenüber (Loneliness is the face you wear). Schließlich ist noch die Frage nach dem Ausdruck von Definitheit in artikellosen Sprachen zu beantworten. Hier kann besonders auf die Untersuchung von Friedrich (2009) hingewiesen werden, die gezeigt hat, dass in transitiven Sätzen des Russischen die Platzierung des Akzents auf Objekt (= indefinit) oder Verb (= definit) in etwa der mit der Artikelwahl gegebenen Differenzierung im Deutschen entspricht.

\subsection{Exhaustive Interrogativsätze und verwandte Exklamativa}

Unser nächstes Beispiel eines dt.-engl. Kontrastes ist meines $\mathrm{W}$ issens noch nie genau untersucht worden und betrifft eine Teilmenge von Fragewortoder Inhaltsfragen, d.h. durch Fragesätze ausgedrückte Fragen, die eine voll- 
ständige Liste von Antworten verlangen. In Interrogativsätzen dieser Art folgt der Quantor alle(s) dem Interrogativpronomen im Mittelfeld und signalisiert, für welche Dimension des Fragesatzes eine Liste als Antwort erwartet wird. Im Deutschen sind solche exhaustiven Interrogativa für jede zulässige Funktion von Interrogativpronomina möglich (Subjekt, direktes oder indirektes Objekt, Genitivattribut, adverbiale Adjunkte). Als Numerus des Verbs ist immer nur der Singular zu beobachten:

(25) a. Wen hast du alles dort gesehen?

b. Wer kommt alles zu dem Treffen?

c. Wo bist du alles/überall gewesen?

d. Wozu braucht man alles ein Auto?

e. Womit kannst du alles umgehen?

f. Wessen Zimmer hast du alles gereinigt?

In vielen Varietäten des Englischen, vor allem im amerikanischen Englisch, weniger aber im britischen Englisch, sind solche Konstruktionen problemlos für Interrogativpronomina in Subjekts- und Objektsposition, allerdings bestenfalls marginal, wenn nicht ausgeschlossen in allen anderen Fällen. Der Quantor folgt stets direkt auf das Interrogativpronomen:

(26) a. What all did you see there?

b. Who all will come to the meeting?

c. ?Where all did you go on your trip?

f. *What all does he need this for?

Die völlig unterschiedliche Verankerung dieser Interrogativkonstruktion im Deutschen und im Englischen zeigt sich jedoch auch in einem weiteren Kontrast. Es ist eine bekannte Tatsache, dass Exklamativsätze in vielen Sprachen auf Interrogativsätzen aufbauen oder sogar davon ableitbar sind. Im Deutschen unterscheiden sich diese Exklamativsätze von Interrogativsätzen durch die Wortstellung (Finalstellung des Verbs) und durch die Intonation. Ein weiterer Unterschied besteht jedoch auch darin, dass als Interrogativpronomina lediglich wie und was fir ein auftauchen, parallel zu bow und what a im Englischen:

(27) a. Wie groß der Junge geworden ist!-How tall this boy is!

b. Was für ein Auftritt (das war)! - What a performance (that was)!

Diese distributionelle Lücke wird durch die mit quantifizierenden Interrogativa verwandten Exklamativsätze gefüllt. Im deutlichen Kontrast zum Englischen haben alle möglichen Interrogativa dieses Typs im Deutschen exklamative Gegenstücke, in denen dann alle Interrogativpronomen möglich sind: 
DEUTSCH: ja (,mit anderer Wortstellung) - ENGLISCH: nein

(28) a. Wer da alles reden wollte! ,The people who wanted to give a speech!c

b. Wen Paul alles kennt! ,The people Paul knows!

c. Wo der alles hinfährt!,(Amazing,) the places he visits!

d. Wozu der alles Geld braucht! (It is amazing) the reasons he needs money for!

e. Wo der alles/überall abgeschrieben hat! ,The texts he copied from!

Wie auch in den unter (29) aufgeführten Beispielen unterscheiden sich diese Exklamativsätze von den entsprechenden Interrogativa durch Verbstellung und Intonation. Die jeweils angefügten Übersetzungen ins Englische exemplifizieren einen Typ von Exklamativkonstruktionen, der wiederum im Deutschen nicht anzutreffen ist: durch Relativsätze modifizierte Nominalphrasen.

\subsection{Kontrastive Beobachtungen als Ausgangspunkt für typologische Untersuchungen: \\ Exklamativsätze als konventionalisierte Insubordination}

Der eben diskutierte Phänomenbereich kann auch als Illustration für die oben angeführte Behauptung herangezogen werden, dass feinkörnige kontrastive Untersuchungen zum Ausgangspunkt für weitergehende typologische Untersuchungen werden können. Verschiedene vergleichende Untersuchungen zu Exklamativsätzen haben gezeigt, dass sich dieser ,Typ' sehr deutlich von den drei Satzmodi unterscheidet, deren Existenz für alle Sprachen angenommen wird. In König/Siemund (2007, S. 316f.) wurden diese Sätze daher zu den ,minor sentence types` gerechnet und nicht mit Deklarativsätzen, Interrogativsätzen und Imperativen als vierte Wahlmöglichkeit im System der Satzmodi gesehen. Es sind vor allem drei Eigenschaften, die den Exklamativsätzen einen völlig anderen Status verleihen:

(i) große Zahl von möglichen Konstruktionen

Im Gegensatz zu den drei genannten wohl in allen Sprachen unterschiedenen Satzmodi können Exklamativsätze eine Vielzahl von Formen annehmen, die bei den ,anderen' Satzmodi nicht zu beobachten ist. Dabei können die zu beobachtenden Formen keinesfalls als Konstruktionen zum Vollzug ,indirekter Sprechakte ' bezeichnet werden, d.h. als Sätze, die ihre exklamative Interpretation erst als Teil der Äußerungsbedeutung in bestimmten Kontexten erhalten. Ihre Verwendung als ,Ausruf ist Teil ihrer konventionellen Satzbedeutung. 
(29) a. Dass ich das noch erleben darf!

b. Wie intelligent diese Frau ist!

c. Was ist diese Frau doch intelligent!

d. Schau dir diese Intelligenz an!

e. Ist diese Frau nicht unglaublich intelligent!

f. Die ist vielleicht intelligent!

g. Und ob (die intelligent ist)!

h. (Es ist erstaunlich), was die alles weiß!

i. Diese Frau ist so intelligent!

j. Diese Wortwahl! Diese Klarheit der Argumentation! Einfach beeindruckend!

(ii) Merkmale von Subordination

Eine weitere bemerkenswerte Eigenschaft ist die Tatsache, dass eine Teilmenge dieser für Exklamationen verwendbaren Konstruktionen deutliche Merkmale von Subordination aufweist. Im Deutschen sind diese Merkmale vor allem Verbendstellung und einleitende Konjunktionen (dass, ob). Insofern sind diese Sätze als eingebetteter Teil von komplexen Sätzen analysierbar oder aber als formal subordinierte Sätze, die als selbstständige Äußerungen verwendet werden.

(iii) Spezialfälle der drei Modi deklarativ, interrogativ, imperativ

In den Fällen, in denen keinerlei Anzeichen für Nebensatzstrukturen gegeben sind $(29 \mathrm{~d}-\mathrm{f})$, handelt es sich um spezielle Verwendungen eines der drei Modi imperativ, interrogativ und deklarativ.

Die Möglichkeit einer vergleichenden Systematisierung solcher Konstruktionen ergibt sich, wenn man sie nicht als Varianten eines 4. Satzmodus, sondern als Manifestationen dessen betrachtet, was Evans (2007) als „Insubordination" bezeichnet hat, d.h. als Resultat eines konventionalisierten Gebrauchs von ursprünglich subordinierten Strukturen als unabhängige Sätze. Ausgangspunkt der Analyse ist dann die Tatsache, dass es sich um Strukturen handelt, die normalerweise nicht in Hauptsätzen, sondern in Nebensätzen auftauchen und in vielfältiger Weise reduziert sein können. In seiner Pionierstudie zu einer Typologie der ,Insubordination ' hat Evans gezeigt, dass solche Konstruktionen vor allem drei Funktionen haben: (a) sie werden als direktive Sprechakte verwendet, was in unseren Beispielen nicht zutrifft, (b) sie drücken bestimmte modale Rahmen aus (deontisch, evidentiell, exklamativ etc.) und (c) sie kennzeichnen verschiedene Diskurskontexte. Ihre historische Entwicklung lässt sich durch die folgenden vier Stufen charakterisieren:

(30) subordinierte Konstruktion $>$ Ellipse des Hauptsatzes $>$ Einschränkung der Interpretation $>$ Reanalyse als unabhängiger Hauptsatz 
Formale Indikatoren des ursprünglich subordinierten Charakters dieser Konstruktionen sind:

(i) Ellipse des Hauptsatzes, sowie weitere Reduzierungen;

(ii) spezielle subordinierte Verbformen, wie der Konjunktiv oder der Infinitiv;

(iii) der Gebrauch von Konjunktionen;

(iv) die für Subordination typische Stellung von Konstituenten.

Aus den eben skizzierten Beobachtungen zum Deutschen ergibt sich auch eine neue Möglichkeit, Exklamativsätze generell einzuordnen. Exklamativsätze sind häufig als parametrisierte Interrogativa (,Wh-questions") bezeichnet und analysiert worden, die bereits die Antwort auf die gestellte Frage enthalten (,self-answering questions"). Und in der Tat enthalten diese Satztypen in einer großen Zahl von Sprachen sehr oft ein Interrogativpronomen. Mit dieser Analyse wird man jedoch den eben skizzierten Eigenschaften, die auch in anderen Sprachen deutliche Parallelen haben, nicht gerecht.

Sehr oft sind sprachvergleichende Untersuchungen, insbesondere im Rahmen der generativen Grammatik, davon ausgegangen, dass der Vergleich auf der Basis übereinzelsprachlicher Kategorien erfolgen kann. Diese Auffassung ist der theoretischen Position des Strukturalismus sowie der generell geübten Praxis einzelsprachlicher Beschreibungen diametral entgegengesetzt, nach denen jede Sprache eine spezifische ,innere Form " aufweist, die es zu beschreiben gilt. In den letzten Jahren haben besonders Lazard und Haspelmath den sprachspezifischen Charakter von Kategorien betont und auf die Unabdingbarkeit hingewiesen, vergleichende Untersuchungen auf „,komparative Konzepte“ zu stützen (vgl. Lazard 2006; Haspelmath 2010). An diesem Punkt kann die Kontrastive Linguistik eine besondere Rolle spielen, indem sie durch feinkörnige vergleichende Untersuchungen mit anderen Sprachen zeigt, in welcher Weise bestimmte Kategorien bei aller Ähnlichkeit und Überlappung mit anderen Sprachen sprachspezifisch sind und oft in ganz spezieller oder sogar einzigartiger Weise Bündel von Eigenschaften verknüpfen. Ein paar Anmerkungen zur Reflexivität im Deutschen, die auch in den Arbeiten des IDS (Zifonun 2003) eine wesentliche Rolle gespielt hat, sollen diese These illustrieren:

(i) Im Gegensatz zum Englischen und den finno-ugrischen Sprachen sind im Deutschen Reflexivpronomen (sich) und Intensifikator (selbst) formal unterschieden und kombinierbar (sich selbst). Im Gegensatz zu diesen Sprachen wird das Reflexivum im Deutschen nicht nur zum Ausdruck für Bindung bzw. Koreferenz verwendet, sondern auch als sog. „middle marker" (Plötzlich öffnete sich die Tür, dieses Buch liest sich sehr gut, Karl kniete sich hin etc.). 
(ii) Im Gegensatz zum Polnischen (sie vs. siebie) und Niederländischen (zich, zichrelf) gibt es im Deutschen nur ein Reflexivum.

(iii) Im Gegensatz zum Englischen ist im Deutschen dieses Reflexivpronomen für die dritte Person (sich) verfügbar und flektiert weder nach $\mathrm{Nu}$ merus noch nach Kasus.

(iv) Im Gegensatz zu den skandinavischen Sprachen (sin) gibt es im Deutschen kein attributiv verwendbares Reflexivum.

(v) Im Gegensatz zum Mandarin ( $\left(i j i^{2}\right.$ ) und zum Japanischen (zibun no) hat das Deutsche kein Reflexivum im Genitiv bzw. in possessiven Kontexten. Was in solchen Kontexten im Deutschen verwendet wird, entspricht genau dem, was beim historischen Prozess der Erneuerung von Reflexiva im Englischen zu beobachten ist: Das Possessivpronomen bzw. die Genitivform des Personalpronomens wird mit dem Intensifikator selbst verbunden, um diese Lücke zu füllen:

(31) Eine Stadt vergewisserte sich ihrer selbst.

(vi) Im Gegensatz zum Englischen kommt das deutsche Reflexirum auch in Adjunkten vor, in denen keinerlei Kontrast ausgedrückt wird (Er hat eine schöne Woche vor sich).

(vii) Im Gegensatz zum Mandarin und zum Lateinischen gibt es keine logophorische Verwendung für das Reflexivpronomen im Deutschen.

(viii) Im Gegensatz zum Englischen wird das deutsche Reflexivum auch in Kombination mit Verben der Körperpflege verwendet (engl. He showered, washed, shaved, dressed carefully - dt. Er duschte sich, wusch sich, rasierte sich, zog sich an).

Diese Liste, die sich noch verlängern ließe, arbeitet durch einen Vergleich prominente Züge des Pronomens sich heraus und kennzeichnet damit in etwa die einzelsprachliche Kategorie ,Reflexivum im Deutschen' in klarer Differenzierung von den entsprechenden deskriptiven Kategorien anderer Sprachen.

\section{Kontrastive Linguistik und Mikrovariation}

Aus den bisherigen Ausführungen ist bereits mehrfach deutlich geworden, dass Kontraste zwischen zwei Sprachen bei genauerer Betrachtung nur für bestimmte Varietäten dieser Sprachen gelten. Solche Einschränkungen sind insbesondere bei Vergleichen mit dem Englischen angebracht, das in seiner globalen Ausbreitung in sehr vielen unterschiedlichen Ausprägungen anzutreffen ist. Insofern ist es auch angebracht, einen Blick auf das Verhältnis und die mögliche Interaktion von Beschäftigung mit zwischensprachlicher Variation und innersprachlicher Variation zu werfen, so wie sie heute unter der Bezeichnung, Mikrovariation' praktiziert wird. Das mit dieser Bezeich- 
nung verbundene Programm unterscheidet sich von traditioneller Dialektologie nicht nur dadurch, dass die Syntax neben der Morphologie im Mittelpunkt der Untersuchung steht, sondern auch durch anspruchsvollere Zielsetzungen, die z.T. den im Rahmen der Typologie verfolgten analog sind: In welchem Rahmen und in welchen Grenzen unterscheiden sich Varietäten einer Sprache voneinander? Warum sind die Unterschiede in manchen Domänen besonders zahlreich (Lokaladverbien, deiktische Systeme, Relativpronomina) und in anderen nicht (grundlegende Anordnung von Konstituenten, Artikelgebrauch)? Gibt es generelle oder sogar universelle Eigenschaften von Nichtstandardvarietäten bzw. Varietäten ohne Traditionen des schriftlichen Gebrauchs? Für viele europäische Sprachen gibt es größere Projekte zur Mikrovariation, die zu einem großen Teil auch an einer Implementierung und Weiterentwicklung des Minimalismusprogramms arbeiten (vgl. Poletto 2000; Obenauer 2006).

In welcher Weise überlappen sich nun die Interessen und Zielsetzungen von Kontrastiver Linguistik und Mikrovariation und inwieweit ist eine wechselseitige Befruchtung möglich? Zunächst einmal interessieren sich beide Programme für Variation, allerdings ist es interlinguale Variation in einem Fall und intralinguale Variation im anderen. Für genetisch eng verwandte Sprachen wird der Unterschied zwischen den beiden Programmen sehr gering sein. Sofern kontrastive Untersuchungen mit der Zielsetzung verbunden sind, praktische Implikationen für Fremdsprachenunterricht zu haben, wird ihr Fokus allerdings auf Standardvarietäten ruhen. Andererseits wird eine solche Einschränkung der Perspektive sicher oft nur auf Kosten eines realistischen Bildes von den Kontrasten zwischen zwei Sprachen möglich sein. Viele der erwähnten Untersuchungen zur Mikrovariation haben gezeigt, dass bestimmte Lücken einer Standardsprache oft durch Phänomene anderer Varietäten gefüllt werden. Insofern liefern Kontrastierungen von zwei Standardsprachen kein vollständiges und auch kein realistisches Bild zwischensprachlicher Kontraste. So zeigen die Untersuchungen zu den Varietäten des Englischen beispielsweise, dass Relativpronomina (who, whose, which etc.) so gut wie nie in Dialekten auftauchen und dort invarianten Partikeln (as, what, that etc.) entsprechen. Andererseits gibt es auch in Varietäten des Deutschen invariante Partikeln (wo, was) anstelle der flektierenden Relativpronomina (vgl. Kortmann (Hg.) 2004; Murelli 2011). Eine für den Unterricht Deutsch als Fremdsprache sicherlich interessante Beobachtung ist die Tatsache, dass viele Varietäten des Deutschen die für germanische Sprachen charakteristische Opposition von ungerundeten und gerundeten Vorderzungenvokalen (i vs. y, e vs. $\varnothing$ etc.) nicht haben. Und, um ein sehr spezielles aber in Rahmen der Generativen Grammatik oft diskutiertes Beispiel zu erwähnen, das Phänomen der ,parasitic gaps“", d.h. der Bezug von einer Füllerkonstituente auf zwei folgende Lücken (vgl. (32a)), gibt es nicht im Hochdeutschen, aber durchaus im Bairischen (32b): 
(32) a. engl. This is the only pub I was ever thrown out of _ for wearing jeans in _.

b. Dieser Ball wenn _ gekommen wäre hätte ich _ gehabt.? (Kontext: Tennisspiel)

Die eben gemachten Beobachtungen berechtigen sicher zu dem Schluss, dass kontrastive Untersuchungen ohne jegliche Berücksichtigung der internen Variation der verglichenen Sprachen nicht immer ein realistisches Bild von den Unterschieden und Gemeinsamkeiten abgeben, und dass in gewissen Fällen Nicht-Standardvarietäten durchaus eine wesentliche Rolle im Fremdsprachenunterricht spielen können. Beobachtungen zu Dialekten können auch dann von besonderem Interesse sein, wenn sie seltene Phänomene betreffen, wenn sie bestimmte Lücken im Spektrum erwartbarer Phänomene füllen oder wenn sie auf Aspekte von früherem oder aktuellem Sprachwandel aufmerksam machen.

\subsection{Gebrauch von attributivem Intensifikator zum Ausdruck von Reflexivität}

Ein bereits erwähntes Phänomen aus dem Bereich Reflexivität verdient in diesem Zusammenhang besondere Erwähnung, die Verwendung von ndl. eigen in verschiedenen Dialekten (u.a. Brabantisch) als Reflexivindikator:

NIEDERLÄNDISCH (u.a. BRABANTISCH)

(33) a. Hij maakt hem/zijn eigen de meesten zorgen over zijn eigen. er macht ihn/ sein eigen die meisten Sorgen über sein eigen ,Am meisten sorgt er sich um sich selbst. ${ }^{c}$

b. Hij wast zijn eigen. ,Er wäscht sich. ${ }^{6}$

Es gibt eine Reihe von z.T. bereits erwähnten Beobachtungen, die diese Verwendung eines ursprünglich possessiven Adjektivs als Reflexivelement plausibel machen:

- Intensifikatoren und Reflexiva sind oft identisch.

- In vielen Sprachen werden die üblichen Intensifikatoren auch attributiv gebraucht (türk. kendi; chines. rijǐ).

- Im Germanischen gibt es einen speziellen attributiven Intensifikator (dt. eigen, engl. omm, norw. egen), der sich aus Ausdrücken für Besitz entwickelt hat.

(34) a. Mein eigenes Haus würde ich nie verkaufen.

b. Sein eigener Tod überraschte uns mehr als der seines Bruders.

- Somit ist es nicht überraschend, dass auch dieser Intensifikator als Reflexivmarkierung benutzt wird, so wie es in Varietäten des Niederländischen der Fall ist.

Auf die schriftliche Repräsentation von bairischer Phonetik wird hier verzichtet. 
5.2 Weitere Beispiele

Zu den besonders interessanten Phänomenen in Varietäten des Englischen gehört auch die Verwendung von self-Formen in Subjektposition im irischen English. Diese Verwendung entspricht nicht traditionellen und typologischen Beobachtungen, nach denen Reflexivpronomina nicht in Subjektposition vorkommen, und ist vor allem mit dem Prinzip A der Bindungstheorie von Chomsky nicht vereinbar, nach dem (reflexive) Anaphern in der Kategorie gebunden sind, die ihre Rektion bestimmt, also grob gesagt nur in Objektpositionen auftauchen. Die folgenden authentischen Beispiele scheinen all diesen Beobachtungen und Prinzipien zu widersprechen:

IRISCHES ENGLISCH

(35) a. It's himself is going to speak today. ,Er selbst wird heute sprechen.

b. Himself was not looking too friendly today. ,Er [z.B. der Boss] schaut heute nicht sehr freundlich drein.

c. And it's himself that told me that up in a pub. (Filppula 1999) ,Und er selbst hat es mir heute in einer Kneipe erzählt."

Die deutschen Übersetzungen zeigen allerdings, dass hier eine Analyse als Reflexivpronomen nicht unbedingt zwingend ist. In (35a, b) handelt es sich um die Identifizierung einer in bestimmten Kontexten zentralen Person. Dieser Zentralitätseffekt ist eine semantische Eigenschaft von Intensifikatoren wie dt. selbst. Für die Beispiele (35a, c) wurde die Übersetzung, Personalpronomen + Intensifikator ' gewählt (er selbst), so dass man hier fragen kann, ob nicht auch im Englischen self-Formen manchmal nicht zum Ausdruck von Koreferenz dienen, sondern kombinatorisch $($ self + bim) zu analysieren und kompositionell zu interpretieren sind. Eine analoge Analyse könnte die Subjekte in (35) einfach als Reduktionen des komplexeren Ausdrucks be bimself auffassen. Andererseits wird hiermit die Frage aufgeworfen, ob nicht in unzulässiger Weise eine Analyse aus der Sicht des Deutschen erfolgt. Diese Überlegungen sollen an dieser Stelle nicht weiterverfolgt werden.

Besonders interessante Phänomene des Deutschen, die auf unterschiedliche Stufen aktueller Sprachwandelprozesse hinweisen, sind z.B. der vieldiskutierte totale Verlust des Präteritums im Süddeutschen und der damit verbundene Verlust des Plusquamperfekts und seine Ersetzung durch das doppelte Perfekt. Die folgenden Beispiele sind aus dem Bairischen, verzichten aber wiederum auf jegliche phonetische Differenzierung vom Hochdeutschen:

(36) a. Wie hat er doch gleich geheißen? (Wie hieß er doch gleich?)

b. Ich habe ihn schon vorher gefragt gehabt. (Ich hatte ihn schon vorher gefragt.)

Diese Beispiele zeigen, dass die Entwicklung einer narrativen Funktion für das deutsche Perfekt im Süden weiter fortgeschritten ist als im Norden und 
auch Hilfsverben und andere Verben erfasst hat, die im Hochdeutschen selten oder nie mit dem Perfekt verbunden werden. Damit ist dann auch das Plusquamperfekt betroffen, das durch das doppelte Perfekt ersetzt wird.

Als abschließendes Beispiel von dialektalen Phänomenen im Deutschen, die auch wegen ihrer theoretischen Implikationen von besonderem Interesse sind, sei noch die Entwicklung der Modalpartikel denn erwähnt. Während diese Partikel im Hochdeutschen vor allem in reaktiven Fragen, d.h. in Fragen, die auf eine vorausgehende Interaktion Bezug nehmen, verwendet wird, ist sie im Bairischen zu einem Frageindikator ohne jegliche kontextuelle Restriktionen geworden (vgl. Bayer ersch.demn.).

\section{Kontrastive Linguistik und interkulturelle Kommunikation}

Die Entwicklung der strukturellen Linguistik seit de Saussure ist u.a. dadurch gekennzeichnet, dass in zunehmendem Maße von jeglicher kontextuellen Einbettung (situativ, textuell, kulturell etc.) abstrahiert wurde und somit das Sprachsystem, die langue oder die Kompetenz zum ausschließlichen Gegenstand der Sprachwissenschaft wurde. Die einzigen kontextuellen Faktoren, die noch berücksichtigt werden, sind somit: die Koordinaten der Sprechsituation, die sozialen Beziehungen zwischen Sprechern und Adressaten, ihre Präferenzen, ihr Status, die Prinzipien kooperativer Kommunikation à la Grice, der unmittelbare konversationelle Kontext und der Stand des konversationellen Austausches, d.h. die Informationsstruktur. In neueren Programmen der Sprachpragmatik und im vergleichenden Programm der interkulturellen Kommunikation werden diese Abstraktionen aufgehoben und an die Stelle des Vergleichs von Sprachsystemen tritt der Vergleich zwischen kommunikativen Normen in Kontexten, zwischen unterschiedlichen Gebrauchsweisen von ähnlichen Mitteln und von Kontrasten bei weitgehender Äquivalenz (Übersetzung). ${ }^{8}$ Das mit der Bezeichnung ,,interkulturelle Kommunikation ${ }^{c c}$ verbundene Programm knüpft zwar z.T. an vergleichende Beobachtungen zu verschiedenen Sprachsystemen an, beschäftigt sich aber im Wesentlichen mit dem Vergleich von Kommunikationsnormen und Kommunikationsverhalten, die bei interkulturellen Begegnungen zu beobachten sind. Die Beschäftigung mit solchen Kontrasten zielt also in besonderem Maße ab auf die Vermittlung von interkultureller Kompetenz über den Erwerb einer Fremdsprache hinaus. Die folgenden Ausführungen sollen einen Eindruck von zentralen Themen dieses Forschungsprogramms vermitteln und mögliche Interaktion, Überlappung aber auch Divergenz zwischen Kontrastiver Linguistik und dem Programm der interkulturellen Kommunikation ansprechen.

Bei Übersetzungsvergleichen, insbesondere bei der Übersetzung kultureller Schlüsselwörter, ist die Notwendigkeit, die Einbettung in verschiedene Kontexte zu berücksichtigen, besonders groß (vgl. Utz 2007). 
Ein vielbeachtetes Thema sprachvergleichender Untersuchungen, das zwar noch auf unterschiedliche Systeme Bezug nehmen, aber darüber hinaus auch unterschiedliche Verwendungen analoger Unterscheidungen in Abhängigkeit vom sozialen und kulturellen Kontext in den Blick nehmen muss, sind die sog. Honorifika. In den europäischen Sprachen gehören dazu besonders die Anredeformen mit Unterscheidungen wie $d u$ vs. Sie (Deutsch), tu vs. vous (Französisch) oder ty vs. yy (Russisch). Aus der Existenz solcher weitgehend paralleler Subsysteme darf man keinesfalls auf parallele Verwendungen schließen. Hier bestehen deutlich unterschiedliche Normen in den erwähnten und anderen Sprachen, die zudem in den letzten Jahrzehnten starken Veränderungen unterworfen waren. In den früher sehr hierarchisch strukturierten Gesellschaften Ostasiens sind diese Differenzierungen noch wesentlich reicher und betreffen nicht nur Anredeformen, sondern auch Bezugnahmen von Sprechern auf sich selbst, Verbformen und sogar die Auswahl von Vokabular je nach sozialer Situation. Da das Inventar der Anredeformen im Japanischen sehr umfangreich ist, werden die vorausgegangenen Beobachtungen zunächst einmal durch verschiedene Wahlmöglichkeiten bei der 1. Person Singular illustriert. Die Wahl eines dieser Ausdrücke hängt davon ab, wer spricht (männlich vs. weiblich) und wer die Gesprächspartner/innen sind.

\begin{tabular}{|l|l|l|l|l|}
\hline & formal & & & informell \\
\hline männlich & watakushi & watashi & boku & ore \\
\hline weiblich & watakushi & watashi & watashi & atashi \\
\hline
\end{tabular}

Tab. 1: Referenz auf den Sprecher im Japanischen (1. Person Singular)

Für europäische Kommunikationsnormen noch erstaunlicher ist die Tatsache, dass als Entsprechungen für das Verb geben' mindestens sechs Wahlmöglichkeiten zur Verfügung stehen, je nach der sozialen Stellung des Empfängers bzw. der Empfängerin und nach der Identität des Gebenden (Sprecher/in oder Adressat/in). Um einen Eindruck von den sozialen Konnotationen dieser Verben zu vermitteln, müsste man das Verb ageru/ agemasu mit den entsprechenden Subjekten, etwa mit, ich biete Ihnen ehrerbietig an' und das Verb kurem/kuremasu mit ,Sie überlassen mir großzügig übersetzen.

Zentraler noch für das Arbeitsprogramm der interkulturellen Kommunikation als diese immer noch in unterschiedlichen Systemen verankerten Kontraste ist der Vergleich von unterschiedlichem Sprachverhalten in vergleichbaren Situationen, in bestimmten institutionellen Rahmen oder ganz generell. Was die speziellste Ebene der vergleichbaren Situationen anbelangt, so bilden vor allem Bedingungen und Ausführungen von Sprechakten ein wesentliches Thema dieser Untersuchungen. Internationale Konferenzen zeigen, dass unterschiedliche Sprachgemeinschaften unterschiedliche ,Kul- 
turen` für die Sprechakte ,Kritik', ,Entschuldigung,, „Einladung', ,Bitte', ,Aufforderung ${ }^{c}$ oder ,Beschwerde ${ }^{c}$ ausgebildet haben. Es entspricht einem weitverbreiteten Eindruck, dass von deutschen Teilnehmern geäußerte Kritik als besonders derb, direkt und manchmal geradezu unhöflich empfunden wird, während Amerikaner selbst deutliche Kritik durch Dankesworte und kurze positive Würdigungen einleiten. Davon, wie man in Japan Selbstkritik übt und sich entschuldigt, haben Berichte über den Reaktorunfall in Fukushima anschaulich Zeugnis abgelegt: Tränen und sich verneigen vor den Zuschauern sind Teil der entsprechenden Rituale. Auf einer allgemeineren Ebene ist es Kommunikation in institutionellen Rahmen, die im Mittelpunkt vergleichender Untersuchungen steht: unterschiedliches Verhalten bei Verhandlungen, unterschiedliche verbale Geschäftskulturen. Hier geht es nicht mehr um die unterschiedliche Ausführung einzelner Sprechakte, sondern um unterschiedliche Normen, Regeln und Konventionen bei Sprechtätigkeiten.

Auf einer noch allgemeineren Ebene geht es um den Vergleich von kulturell distinktiven Registern oder Sprechstilen. Ein besonders vielbeachtetes und vielzitiertes Beispiel ist hier dugri speech (straight talk), der Sprechstil der in Israel geborenen Sabras, der als besonders direkt, offen, derb, „zum Thema" charakterisiert wird. Durch seine Vernachlässigung aller Rücksichtnahme auf Höflichkeit und Reibungslosigkeit interpersonaler Beziehungen ist dieser Stil dem der arabischen Nachbarn (musayra) völlig entgegengesetzt.

\section{Zusammenfassung}

Nach den vorausgegangenen Versuchen, der Kontrastiven Linguistik einen Platz im Spektrum verschiedener Ansätze zur vergleichenden Sprachanalyse zuzuweisen, sind wir nun in der Lage, die wesentlichen Punkte der Agenda einer Kontrastiven Linguistik zusammenzufassen. Für die KL ergibt sich abschließend das folgende Profil:

\section{a) Orientierung}

Die Kontrastive Linguistik hat primär eine synchrone Orientierung, auch wenn sich bei genetisch verwandten Sprachen und bei Einbeziehung von Varietäten interessante historische Probleme ergeben können. Hier sind besonders die erwähnten Phasenverschiebungen beim Sprachwandel in genetisch verwandten Sprachen von großem Interesse für die Suche nach Verallgemeinerungen im Sprachwandel.

b) Granularität

Zielsetzung der Kontrastiven Linguistik sollte es sein, umfassende und feinkörnige Vergleiche von Sprachpaaren zu erstellen, die typologischen Verallgemeinerungen nicht unbedingt zugänglich sind. Insofern sind kontrastive Analysen ein Komplement zur Typologie und nicht eine Typologie in 
kleinem Rahmen. Diese Betonung von Feinkörnigkeit bedeutet jedoch nicht, dass die Betonung auf Einzelbeobachtungen und nicht auf Verallgemeinerungen liegt, aber diese Verallgemeinerungen betreffen die zu vergleichenden Sprachen und haben mit den typologischen Generalisierungen nur insofern etwas zu tun, als letztere den Orientierungsrahmen für einen kontrastiven Vergleich bereitstellen.

c) Skopus

Kontrastive Analysen sollten sich im Wesentlichen auf einen bilateralen Sprachvergleich beschränken, auf den Vergleich zwischen Mutter- und Fremdsprache, zwischen Erst- und Zweitsprache, zwischen Ausgangs- und Zielsprache, je nachdem, welche praktischen Implikationen ins Auge gefasst werden. Eine Ausweitung über diesen engen Skopus hinaus ist nur dann sinnvoll, wenn die Zielsetzung, umfassende vergleichende Grammatiken zu schreiben, zugunsten von arealen Studien oder typologischen Pilotstudien aufgegeben wird. Kleinere Ausschnitte von Sprachen kann man auch für drei, vier oder fünf Sprachen vergleichen und aus der Sicht einer dritten Sprache kann man interessante Beobachtungen über zwei andere machen. Es ist aber genau diese Beschränkung auf zwei Sprachen, die es möglich macht, eine Vielzahl von Parametern der Variation zu untersuchen und sich dem Ziel einer holistischen Typologie zumindest anzunähern. Die Frage, welche Sprachen sinnvollerweise zu vergleichen sind, wird in allen oben genannten Spielarten des Sprachvergleichs unterschiedlich beantwortet.

d) Perspektive

Mit einer kontrastiven Analyse ist kein besonderer theoretischer Rahmen oder Ertrag verbunden. Kontrastiv beschreiben heißt, eine Sprache aus der Sicht einer anderen zu analysieren und auf diese Weise Eigenschaften zu finden, die sonst nicht ohne Weiteres sichtbar würden. Mit anderen Worten: Die Kontrastive Linguistik hat einen hohen heuristischen Wert für die Entdeckung sprachspezifischer Eigenschaften. Unterschiedliche Vergleichssprachen ergeben unterschiedliche Perspektiven und können verschiedene Eigenschaften erhellen. Eine kontrastive Untersuchung, die nicht zu neuen Einsichten führt, ist sinnlos.

e) Theoretischer Rahmen

Die wahre Herausforderung für kontrastive Analysen besteht darin, Unterschiede zwischen zwei Sprachen in maximal genereller und falsifizierbarer Weise zu beschreiben, nicht in der Wahl eines theoretischen Rahmens. Das Explanandum sind die Kontraste zwischen zwei Sprachen. Kontrastive Analysen, die mit der Absicht bestimmter praktischer Implikationen geschrieben werden, sollten allzu technischen Jargon formaler Modelle möglichst vermeiden. 


\section{Literatur}

Aarts, Flor (1981): The contrastive analysis debate: problems and solutions. In: Studia Anglica Posnaniensia XIV, S. 30-47.

Alatis, John E. (Hg.) (1968): Contrastive linguistics and its pedagogical implications. Georgetown.

Bayer, Josef (ersch.demn.): From modal particle to interrogative marker: A study of German, denn'. In: Brugè, Laura et al. (Hg.): Functional Heads. (= Oxford Studies in Comparative Syntax; The Cartography of Syntactic Structures 7). Oxford.

Evans, Nicholas (2007): Insubordination and its uses. In: Nikolaeva, Irina (Hg.): Finiteness. Theoretical and Empirical Foundations. Oxford u.a., S. 366-431.

Evans, Nicholas (2011): Semantic typology. In: Song, Sae Jung (Hg.): The Oxford handbook of linguistic typology. Oxford u.a., S. 504-533.

Evans, Nicholas/Levinson, Stephen (2009): The myth of language universals. In: Behavioral and Brain Sciences 32, S. 429-492.

Faltz, Leonard M. (1985): Reflexivization: a study in universal syntax. New York u.a.

Filppula, Markku (1999): The grammar of Irish English: language in Hibernian style. (= Routledge Studies in Germanic Linguistics 5). London u.a.

Friedrich, Svetlana (2009): Definitheit im Russischen. (= Potsdam Linguistic Investigations 4). Frankfurt a.M. u.a.

Haspelmath, Martin (2010): Comparative concepts and descriptive categories in crosslinguistic studies. In: Language 86, 3, S. 663-687.

Haspelmath, Martin et al. (Hg.) (2005): The world atlas of language structures. Oxford u.a.

Hawkins, John A. (1986): A comparative typology of English and German: unifying the contrasts. London/Sydney.

Hawkins, John A./Filipović, Luna (ersch.demn): Criterial features in L2 English. Specifying the reference levels of the Common European Framework. Cambridge.

Heine, Bernd/Kuteva, Tania (2005): Language contact and grammatical change. Cambridge u.a.

Hüning, Matthias et al. (Hg:) (2006): Nederlands tussen Duits en Engels. Handelingen van de workshop op 30 september en 1 october 2005 aan de Freie Universität Berlin. Leiden.

König, Ekkehard (1996): Kontrastive Grammatik und Typologie. In: Lang, Ewald/Zifonun, Gisela (Hg.): Deutsch typologisch. (= Jahrbuch des Instituts für Deutsche Sprache 1995). Berlin/New York, S. 31-54.

König, Ekkehard/Gast, Volker (2006): Focused expressions of identity - a typology of intensifiers. In: Linguistic Typology 10, 2, S. 223-276.

König, Ekkehard/Gast, Volker (2009): Understanding English-German contrasts. 2., neu bearb. Aufl. (= Grundlagen der Anglistik und Amerikanistik 29). Berlin. 
König, Ekkehard/Siemund, Peter (2007): Speech Act Distinctions in Grammar. In: Shopen, Timothy ( $\mathrm{Hg}$.): Language typology and syntactic description. Bd. 1: Clause structure. Cambridge, S. 276-324.

König, Ekkehard/Vezzosi, Letizia (2008): Possessive adjectives as a source of intensifiers. In: Seoane, Elena/Lopez-Couso, María José ( $\mathrm{Hg}$.): Theoretical and empirical issues in grammaticalization. 2 Bde. (= Typological Studies in Language 77). Amsterdam u.a., S. 183-206.

Koptjevskaja-Tamm, Maria/Vanhoeve, Martine/Koch, Peter (2007): Typological approaches to lexical semantics. In: Linguistic Typology 11, S. 159-85.

Kortmann, Bernd (Hg.) (2004): Dialectology meets typology. Dialect grammar from a cross-linguistic perspective. (= Trends in Linguistics, Studies and Monographs 153). Berlin/New York.

Kufner, Herbert L. (1962): The grammatical structures of English and German. Chicago.

Lazard, Gilbert (2006): La quêtes des invariants interlangues. La linguistique est-elle une science? (= Bibliothèque de Grammaires et de Linguistique 23). Paris.

Murelli, Adriano (2011): Relative constructions in European non-standard varieties. (= Empirical Approaches to Language Typology 50). Berlin.

Obenauer, Hans-Georg (2006): Special interrogatives, left periphery, Wh-doubling and (apparently) optional elements. In: Doetjes, Jenny/González, Paz (Hg.): Romance languages and linguistic theory 2004. (=Amsterdam Studies in the Theory and History of Linguistic Science: Series 4, Current Issues in Linguistic Theory 278). Amsterdam u.a., S. 247-274.

Plank, Frans (1984a): The modals story retold. In: Studies in Language 8, S. 305-364.

Plank, Frans (1984b): Verbs and objects in semantic agreement. Minor differences that might suggest a major one. In: Journal of Semantics 3, S. 305-360.

Poletto, Cecilia (2000). The higher functional field: evidence from Northern Italian dialects. Oxford/New York.

Reis, Marga/Wöllstein, Angelika (2010): Zur Grammatik (vor allem) konditionaler V1Gefüge im Deutschen. In: Zeitschrift für Sprachwissenschaft 29, S. 111-179.

Rohdenburg, Günter (1990): Aspekte einer vergleichenden Typologie des Englischen und Deutschen. Kritische Anmerkungen zu einem Buch von John A. Hawkins. In: Gnutzmann, Claus (Hg.): Kontrastive Linguistik. (= Forum angewandte Linguistik 19). Frankfurt a.M. u.a., S. 133-152.

Stiebels, Barbara (1996): Lexikalische Argumente und Adjunkte. Zum semantischen Beitrag von verbalen Präfixen und Partikeln. (= Studia grammatica 39). Berlin.

Utz, Peter (2007): Anders gesagt, autrement dit, in other words. München.

van den Nest, Daan (2010): Emergenz und Grammatikalisierung von V1-Konditionalen. Ein Rekonstruktionsversuch am Beispiel des Deutschen und Englischen. Diss., Univ. Gent.

van Haeringen, Coenraad B. (1956): Nederlands tussen Duits en Engels. 2. Aufl. Den Haag. 
Vanhoeve, Martine (Hg) (2008): From polysemy to semantic change. Towards a typology of lexical semantic associations. (= Studies in Language Companion Series 106). Amsterdam u.a.

Wienold, Götz (1973): Die Erlernbarkeit der Sprachen. München.

Zifonun, Gisela (2003). Grammatik des deutschen im europäischen Vergleich. Das Pronomen Teil II: Reflexiv- und Personalpronomen. (= amades 1/03). Mannheim. 